\title{
A PHYSICAL PICTURE FOR THE INTERNAL FRICTION DUE TO DISLOCATIONS AND ATOMIC-DEFECT - DISLOCATION INTERACTIONS
}

A. SEEGER

Max-Planck-Institut für Metallforschung. Institut für Physik, and Universitat stuttgart, Institut für Theoretische und Angewandte Physik, Postfach 800665, D-7000 stuttgart 80, F.R.G.

\begin{abstract}
ABSIRACT
Since the introduction of the "pinned-string model" by J.S. Koehler and its subsequent elaboration by K. Lücke, A. Granato and others, the pinning concept has been used extensively in the interpretation of internal friction experiments involving dislocations. The ongoing refinements of the experiments have made it highiy desirable to put the intuitive pinned-string model on a firmer theoretical basis, to delineate its limitations, and to replace it by more general or better founded concepts where necessary. Among them is the idea that at low temperatures the movement of dislocations should be described in terms of the stress-assisted generation of kink pairs and the drift of kinks along the dislocation lines (in the case of screw dislocations in refractory bcc metals up to temperatures well above room temperature), and the realization that as a rule - the range of the interaction between atomic defects (foreign atoms, self-interstitials, vacancies) and dislocations exceeds considerably the extension of the disiocation cores.

The physical basis and the general features of a theory allowing for the above-mentioned generalizations will be outlined. The theory is necessarily more complicated than the pinned-string model but covers a much wider range of phenomena. It possesses a higher predictive power and may therefore be subjected to more stringent experimental tests. Over the last few years a number of special cases have been worked out by $T$. Ogurtani and the author. For some selected examples results will be quoted and compared with experiments.
\end{abstract}

